\title{
Simulation of Water Drops on a Surface
}

\author{
Eren Algan, Mustafa Kabak, Bulent Ozguc, Tolga Capin \\ Bilkent University, Faculty of Engineering, Department of Computer Engineering, Ankara, Turkey
}

\begin{abstract}
We have designed and implemented a model for rendering and animating water drops moving on a surface under physical effects such as gravity, surface affinity and wind. For improved rendering, the output of the simulation (i.e. the animation sequence of the drops) is recorded for later offline image generation.
\end{abstract}

\section{INTRODUCTION}

Water is one of the natural phenomena which is difficult to model mathematically. The equations that define the statics and dynamics of water involve advanced mathematical constructions, and still they do not apply to water at every scale. In this project, we aimed at rendering water at the droplet scale. In the rest of the paper, we will investigate the commonly used models to represent water drops, the physical phenomena taken into account, and the methods used to render the droplets; along with our choices and contributions.

\section{SURFACE AND DROP MODELS}

In the studies that focus on rendering droplets, generally the position and the shape of the droplets are separated $[1][2][3]$. The separation means that while one model calculates the positions of the drops as discrete entities, another model calculates the shape of the droplet. This is not the case with more general liquid models, in which the liquid body may be a continuous volume. The separation of position and shape allows interchanging one of the models, e.g. one shape model for real-time rendering and one for more realistic rendering [2].

In this project, we implemented a two-level discrete surface model to calculate the positions of droplets, in which the surface is made up of a rectangular grid of cells. Each high-level cell has an affinity property, which defines how much that cell likes water. A droplet will always be on one of the cells, traveling to another if the position calculation turns out to be so. This high-level grid model for the surface is the one used in [1] for flat rectangular surfaces, and in [2] for biparametric Bezier patches.

Whenever a droplet is calculated to move from one cell to another, a low-level, finer grid is imposed on the route of the droplet. This finer grid makes a more detailed rendering of droplets possible. The advancing front of the droplet will not always be in a round shape, it will be affected from the surface impurities. To our knowledge, none of the studies that use the discrete surface model makes any effort to take into account the variation of forces and surface characteristics within the scale of a droplet size.

\section{PHYSICAL PHENOMENA}

Accurate simulation of water motion involves advanced physics. In [4], to calculate the surface tension on the drop and between the drop and the surface, Navier-Stokes equation (which involves partial differentials) is used. Many of the studies simplify the physics. Some models involve only Newtonian dynamics. Our physical model is a modified

3DTV-CON'08, May 28-30, 2008, Istanbul, Turkey 
version of the model described in [2], which is such a model.

\subsection{Forces and Parameters}

The physical forces and physical characteristics that we take into account are:

- Gravity

- Wind

- Surface tension (To give a realistic shape to droplets)

- Interfacial tension between liquid and solid (as a resistive force, like friction)

- Affinity of surface to water (whether the surface is hydrophobic or hydrophilic)

The parameters we ignore are:

- Viscosity of liquid

- Air resistance

\section{MOTION ALGORITHM}

As mentioned above, gravity is taken into account for the simulation. Our model assumes that the direction of the gravity does not change (i.e. position and orientation of the surface is fixed during the simulation) and furthermore, it is parallel to $y$-axis of the surface. Owing to these simplifying assumptions, the motion calculations are reduced to deciding whether a drop moves down or stays where it is in each simulation step (Algorithm 1).

However, if the entire drops do was moving down, the results would not look realistic. Water drops do not always move directly in the direction of gravity: They meander to make streams that do not follow a line on the surface. In order to model this behavior, the drops are allowed to move diagonally to down-left or down-right [1]. Revising the statement about motion calculations above: The motion calculations involve: (1) deciding whether a drop moves down and (2) if it moves, deciding which cell it moves to.

Whenever a drop moves down, it leaves a small amount $r$ of water at its originating cell.

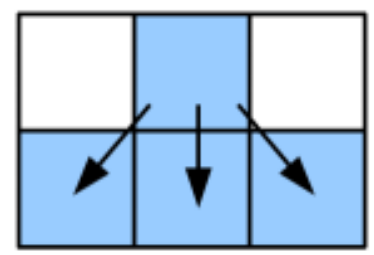

Figure 1 - Possible destinations for a drop.

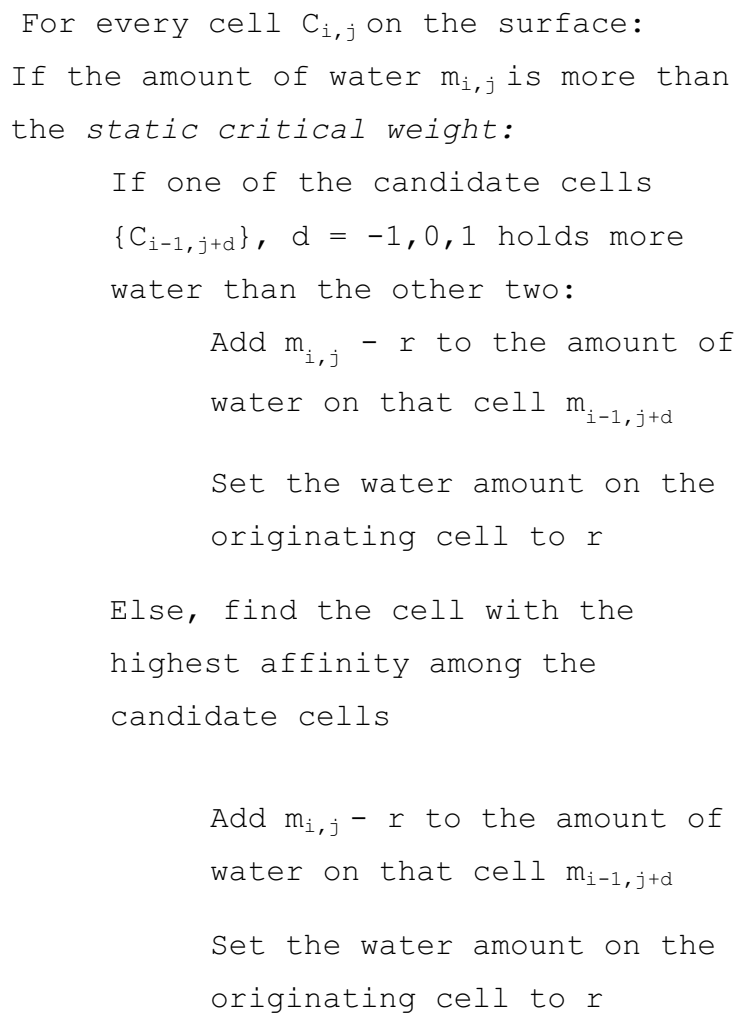

Algorithm 1 - Motion Calculation

\subsection{Decision to Move or Stay}

A drop of water moves down if its weight exceeds a 
configured static critical weight, and continues moving down until its weight is below a configured dynamic critical weight [1]. This method may remind the way a solid object reacts to friction and an external force parallel to the surface it is standing on.

\subsection{Decision of Movement Direction}

Once a drop on cell $\mathrm{C}_{\mathrm{i}, \mathrm{j}}$ ( $\mathrm{i}$ being the row number ascending upwards, $\mathrm{j}$ being the column number) is decided to move down, a routine is invoked to determine the destination cell among the three alternatives $C_{i-1, j-1}, C_{i-1, j}, C_{i-1, j+1}$ (Figure 1). This routine decides the direction as follows [1]:

1. The water amounts at the three cells are compared. The drop moves to the cell which holds the greatest amount of water.

2. If the cells are dry, then the affinity values are compared. The drop moves to the cell which likes water the most.

\section{RENDERING OF DROPS}

Depending on the constraints of an application, various methods can be used to render a water drop. One study that aims real-time rendering in 1993 [1] uses nothing more complicated than a sphere as the shape of the droplets.
Another study [5] uses ellipses to give the appearance of drops deforming due to gravity. More complicated methods include triangle meshes [3], metaballs [2], metaballs with vector fields instead of scalar fields [6] and other implicit surface methods which are used together with particle systems [7].

We used a set of metaballs to render each water drop: A bigger blob is placed in the lower part, and a smaller and flatter blob is placed in the upper part. Thus, the appearance of a drop under the effect of gravity is conveyed.

Using more than one metaball component for each drop allowed us to show the effect of gravity and speed on the drops more realistically. This method also allows the manipulation of drop shape in accordance with surface impurities (See 2. Surface and Drop Models).

The simulation outputs the files that describe the drops as metaball combinations, as described above. Then these files are given to POV-Ray ray tracing software and frames are rendered. In order to obtain the animation, these rendered frames are made to a video. The sequences of drops merging are shown in Figures 2 and 3. Even though the physics of water droplets are used minimally, very realistic animations are obtained that can be used in highly interactive environments such as games.

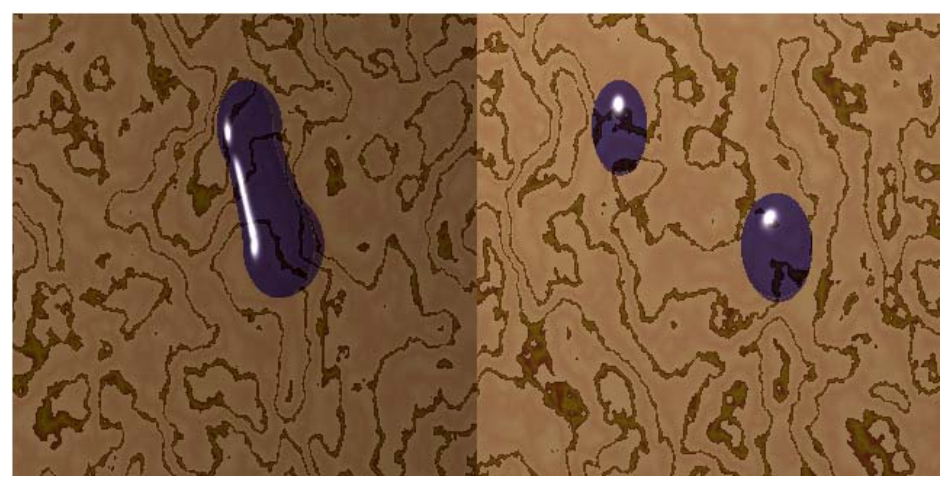

Figure 2 - Results (Merged and individual drops) 

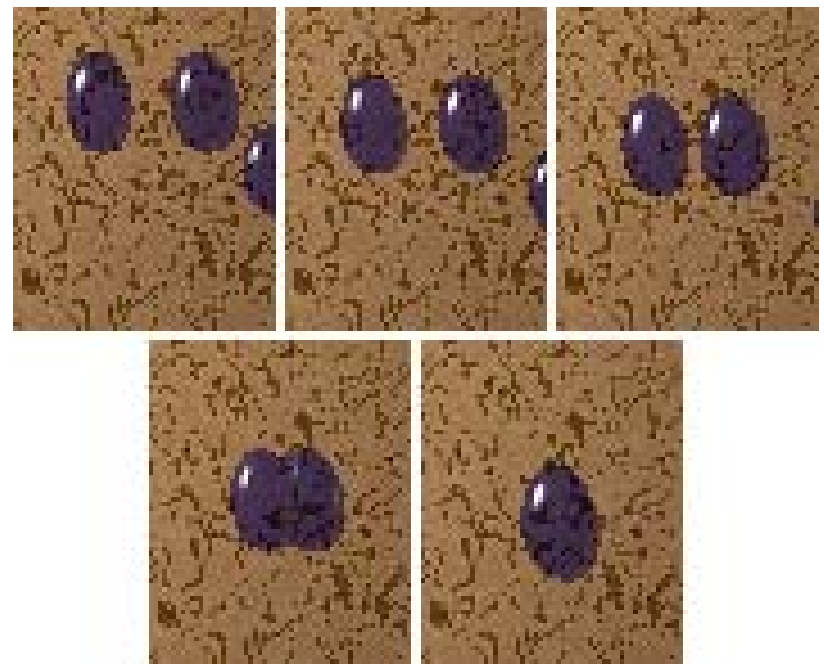

Figure 3 - Merging of Drops

\section{ACKNOWLEDGEMENTS}

This work is supported by European Union 6th Framework Program under Grant No. FP6-511568 (3DTV NoE Project).

\section{REFERENCES}

[1] K. Kaneda, T. Kagawa, H. Yamashita: "Animation of
Water Droplets on a Glass Plate," Proc. Computer Animation' 93, 177-189, 1993.

[2] K. Kaneda, Y. Zuyama, H. Yamashita, T. Nishita: "Animation of Water Droplet Flow on Curved surfaces," Pacific Graphics' 96, 50-65, 1996

[3] P. Fournier, A. Habibi, P. Poulin, "Simulating the Flow of Liquid Droplets," Proceedings of Graphics Interface’ 98, 133-142, 1998

[4] H. Wang, P.J. Mucha, G. Turk, "Water drops on surfaces," Proceedings of ACM SIGGRAPH' 05, 921-929, 2005

[5] S. Djurcilov, "Animation of Water Dripping on Geometric Shapes and Glass Planes," http://www.cse.ucsc.edu/ suzana/water_droplets.ps

[6] Y.J. Yu, H.Y. Jung, H.G. Cho, "A New Rendering Technique for Water Droplet using Metaball in the Gravitation Force," Proceedings of CIKM-98, 7th ACM International Conference on Information and Knowledge Management, 1998

[7] A Murta, J Miller, "Modelling and rendering liquids in motion," Proceedings of WSCG, 194-201, 1999 